REVISTA DE GEOCIÊNCIAS DO NORDESTE

Northeast Geosciences Journal

v. $7, \mathrm{n}^{\circ} 2(2021)$

ISSN: $2447-3359$

\title{
ANÁLISE DA ASSOCIAÇÃO ENTRE VARIÁ VEIS METEOROLÓGICAS E AS INTERNAÇÕES HOSPITALARES POR DENGUE NO MUNICÍPIO DE RIO BRANCO/AC
}

\author{
Beatriz de Souza Freitas ${ }^{1}$; Laura dos Santos \\ Lima $^{2}$; Ana Carla dos Santos Gomes ${ }^{3}$; Lucas Vaz \\ Peres $^{4}$; Alex Santos da Silva ${ }^{5}$
}

${ }^{1}$ Graduanda em Ciências Atmosféricas, Instituto de Engenharia e Geociências, Universidade Federal do Oeste do Pará (UFOPA), Santarém/PA, Brasil.

ORCID: https://orcid.org/0000-0002-8576-9120

Email: beaszfreitas@gmail.com

${ }^{2}$ Graduanda em Ciências Atmosféricas, Instituto de Engenharia e Geociências, Universidade Federal do Oeste do Pará (UFOPA), Santarém/PA, Brasil.

ORCID: https://orcid.org/0000-0002-9356-8409

Email: santoslima1083@gmail.com

${ }^{3}$ Doutora em Ciências Climáticas, Instituto de Engenharia e Geociências, Universidade Federal do Oeste do Pará (UFOPA), Santarém/PA, Brasil.

ORCID: https://orcid.org/0000-0001-7499-8342

Email: anacarlags02@gmail.com

${ }^{4}$ Doutor em Meteorologia, Instituto de Engenharia e Geociências, Universidade Federal do Oeste do Pará (UFOPA), Santarém/PA, Brasil.

ORCID: http://orcid.org/0000-0002-5612-5991

Email: lucasvazperes@gmail.com

${ }^{5}$ Doutor em Meteorologia Aplicada, Instituto de Engenharia e Geociências, Universidade Federal do Oeste do Pará (UFOPA), Santarém/PA, Brasil.

ORCID: https://orcid.org/0000-0002-6365-5659

Email: alex.meteorologia@gmail.com

\section{Resumo}

O presente trabalho avalia a relação da precipitação, umidade relativa, temperaturas mínima e máxima do ar com a ocorrência de internações hospitalares por dengue no município de Rio
Branco/AC, durante o período de 2008 a 2018. Os dados meteorológicos foram obtidos do Banco de Dados de Ensino e Pesquisa, pertencente ao Instituto Nacional de Meteorologia. Já a aquisição dos dados epidemiológicos, deu-se junto ao banco de dados do Departamento de Informática do Sistema Único de Saúde (DATASUS). A partir do teste de Mann Kendall, verificou-se tendência significativa decrescente nos casos de dengue. Em se tratando da correlação cruzada, a única variável que apresentou associação estatística com a dengue foi a umidade relativa, uma vez que durante todo o ano esta apresenta valores que são favoráveis para proliferação e longevidade do vetor. Os resultados mostram que as variáveis de precipitação e temperatura do ar podem subsidiar atividades específicas de prevenção e mitigação ao Aedes Aegypti.

Palavras-chave: Clima; Saúde; Aedes Aegypti.

\section{ANALYSIS OF THE ASSOCIATION BETWEEN METEOROLOGICAL VARIABLES AND DENGUE HOSPITALIZATIONS IN RIO BRANCO CITY/AC}

\section{Abstract}

The present work evaluates the relationship of precipitation, relative humidity, minimum and maximum air temperatures with the occurrence of hospitalizations for dengue in Rio Branco city / AC, during the period from 2008 to 2018 . The meteorological data were obtained from the Database of Teaching and Research, belonging to the National Institute of Meteorology. On the other hand, the acquisition of epidemiological data have been gotten from the database of the Department of Informatics of the Unified Health System (DATASUS). From the Mann Kendall test, there was a significant decreasing trend in dengue cases. When it comes to cross-correlation, the only variable that has a statistical association with dengue was relative humidity, since throughout the year it has values that are favorable for vector proliferation and longevity. The results show that precipitation and air temperature variables can support specific activities to prevent and mitigate Aedes Aegypti.

Keywords: Climate; Health; Aedes Aegypti. 


\section{ANÁLISIS DE LA ASOCIACIÓN ENTRE DIVERSAS ENFERMEDADES METEOROLÓGICAS INTERNACIONALES DEL DENGUE EN LA CIUDAD DE RIO BRANCO / AC}

\section{Resumen}

El presente trabajo evalúa la relación de la precipitación, la humedad relativa, las temperaturas mínimas y máximas del aire con la ocurrencia de hospitalizaciones por dengue en el municipio de Río Branco/AC, durante el período de 2008 a 2018. Los datos meteorológicos se obtuvieron de la Base de Datos de Enseñanza e Investigación, perteneciente al Instituto Nacional de Meteorología. La adquisición de datos epidemiológicos se obtuvo de la base de datos del Departamento de Informática del Sistema Único de Salud (DATASUS). La prueba de Mann Kendall mostró una tendencia significativa a la baja en los casos de dengue. En términos de correlación cruzada, la única variable que mostró asociación estadística con el dengue fue la humedad relativa, ya que a lo largo del año presenta valores favorables para la proliferación y longevidad del vector. Los resultados muestran que las variables de precipitación y temperatura del aire pueden subvencionar actividades específicas de prevención y mitigación del Aedes Aegypti.

Palabras clave: Clima; Salud; Aedes Aegypti.

\section{INTRODUÇÃO}

No Brasil, por ser um país de clima tropical, o índice de doenças vetoriais vem aumentando a cada ano, onde populações são desprovidas de informações, condições sociais e ambientais e vivem em condições sanitárias precárias. Estes fatores associados as mudanças climáticas vem contribuindo para o elevado índice de doenças causada por vetores. (BARACHO, 2013). Existe um potencial epidêmico relacionado à dengue, por ser considerada a doença tropical com maior velocidade de propagação (VIANA et al., 2013).

O número de notificações de dengue no Brasil aumentou drasticamente desde os anos 1980. A rápida urbanização e o não planejamento das cidades, condições de vida precárias, ineficiência da vigilância e do controle do vetor são alguns dos fatores relacionados à dispersão do vírus da dengue. A dengue é uma preocupação global, e as tendências são de rápida expansão da distribuição geográfica do vetor e dispersão do virus (FERREIRA et al., 2018).

O Aedes Aegypti é uma espécie tropical e subtropical, encontrada predominantemente entre as latitudes $35^{\circ} \mathrm{S}$ e $35^{\circ} \mathrm{N}$, com algumas raras ocorrências nas latitudes próximas a $45^{\circ} \mathrm{N}$, durante o verão (FUNASA, 2001). A patologia é designada como uma infecção aguda sistêmica e de etiologia viral que é transmitida pela fêmea do mosquito Aedes Aegypti. A dengue é atualmente a arbovirose mais prevalente no mundo, com cerca de $40 \%$ da população em risco. (VIANA; IGNOTTI, 2013).

A incidência do vírus é influenciada por diversos fatores, tais como: mudanças climáticas, aumento populacional, urbanização, condições sanitárias, socioeconômicas e outros (MENDONÇA et al., 2009). Atinge principalmente as regiões tropicais, que são as mais afetadas devido à taxa de variação pluviométrica e ao aumento da temperatura do ar, fatores que permitem a incubação e proliferação do mosquito transmissor (MONDINI et al., 2009). As alterações climáticas causam impactos no crescimento de 2 bilhões de pessoas sujeitas a dengue. Simulações sugerem que para 2085, aproximadamente de 5 a 6 bilhões de pessoas (50 a $60 \%$ da população global) poderão estar sob risco da transmissão da doença (HALES et al., 2002).

A questão socioeconômica da região influencia nesse módulo por não disponibilizar o saneamento adequado. Assim, a população é obrigada a usar depósitos para armazenar água que poderá servir como criadouros para o Aedes Aegypti (NETO et al., 2019). Na Amazônia, encontram-se nessa situação os estados do Pará, Amazonas, Mato Grosso, Rondônia, e o Acre, cujas populações pobres, além de submetidas à situação geral da divisão em classes sociais, sofrem também diretamente a sazonalidade bioclimática anual, durante as duas estações do ano, chuvosa e seca; caracterizadas pelas enchentes, desmatamentos e queimadas, bem como por surtos de malária, dengue e de doenças respiratórias (DUARTE e MASCARENHAS, 2007).

Estudos anteriores mostram um padrão sazonal de incidência do vírus da dengue coincidente com o verão, devido a maior ocorrência de chuvas e aumento da temperatura do ar (ANDRADE, 2003; BARBOSA, 2007; SOUSA, 2005). Estudos de Souza-Santos (1999) mostraram associações significativas entre a incidência de casos de dengue e umidade relativa, o estudo concluiu que nos meses em que foram verificados maiores índices de umidade relativa do ar, foram notadas as maiores médias de número de criadouros positivos.

Em meados de 1981-1982, diagnósticos de laboratórios brasileiros confirmaram 11.000 casos de dengue no município de Boa Vista, em Roraima (SCHATZMAYR, 2000). Atualmente, estima-se que a dissipação do vírus do Aedes Aegypti já atingiu todas as 27 unidades federativas do país, porém nem todos os municípios foram afetados (CONASS, 2019). A princípio, as variações meteorológicas e socioeconômicas do munícipio soam como fatores agravantes ao desenvolvimento da doença.

Os estudos relacionados à dengue têm se tornado cada vez mais relevantes, não só para o setor saúde, mas também para todos os setores que buscam apoiar e fortalecer a redução dos riscos e agravos relacionados à doenças e que buscam um crescimento maior de diálogos entre setores, operacionalizando parcerias e articulando saberes e experiências, contribuindo para a solução dos problemas encontrados, fazendo com que o sistema de saúde seja mais efetivo (LOPES, 2015). O objetivo principal do estudo é verificar a associação entre as condições meteorológicas e as internações hospitalares por dengue no período de 2008 à 2018.

\section{METODOLOGIA}

\section{1. Área de estudo}

O estado do Acre, localizado no sudoeste da Amazônia (07 $07^{\prime} \mathrm{S}$ : $11^{\circ} 08^{\prime} \mathrm{S}$; $66^{\circ} 30^{\prime} \mathrm{O}$ : $\left.74^{\circ} \mathrm{O}\right)$, apresenta uma superfície territorial de 164.221,36 km², correspondendo a 4\% da Amazônia brasileira e $2 \%$ do território nacional. Possui uma extensão territorial de $445 \mathrm{~km}$ no sentido Norte-Sul e $809 \mathrm{~km}$ entre seus extremos Leste-Oeste. O Acre faz fronteira internacional com o Peru e a Bolívia e, tem divisas estaduais com os Estados do Amazonas e de Rondônia. Sua população é de cerca de 733.559 
mil habitantes, sendo que $66 \%$ da população concentra-se na zona urbana (IBGE, 2010; ACRE, 2010).

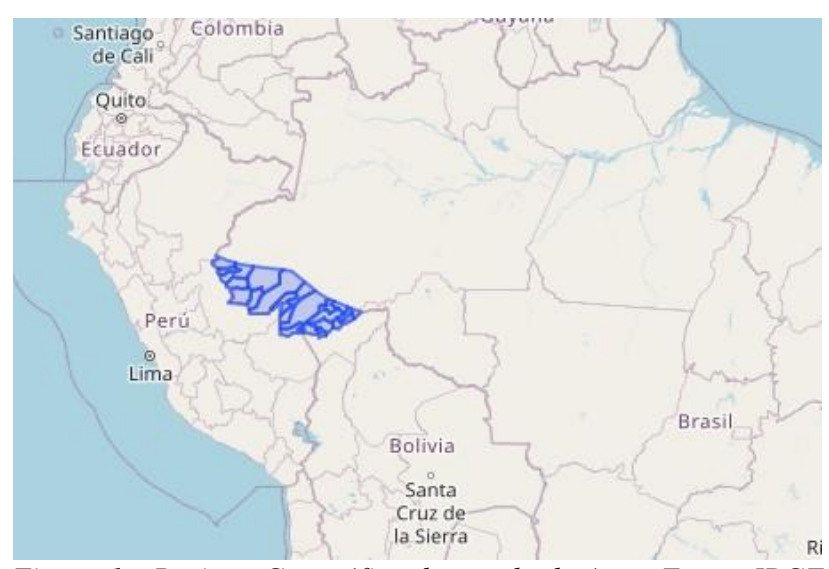

Figura 1 - Posição Geográfica do estado do Acre. Fonte: IBGE (2018).

O município de Rio Branco é a capital do estado do Acre e está localizada na Amazônia Ocidental a 152,5 m acima do nível do mar. Pertence à mesorregião do Vale do Acre, a nordeste do Estado ( $9^{\circ} 30^{\prime} \mathrm{S} ; 67^{\circ} 30^{\prime} \mathrm{O}$ : 69³0'O). A região leste do Estado, é a mais favorecida em termos de infraestrutura administrativa, dos serviços de saúde e de outros setores relevantes da economia regional (ACRE, 2010). A extensão territorial do município é de $8.835,541 \mathrm{~km}^{2}$, correspondendo à $6,5 \%$ do território do estado do Acre.

Tabela 1 - Municípios mais populosos do estado do Acre. Fonte: IBGE (2019). Organização: os autores (2021).

\begin{tabular}{llll}
\hline \hline Ranque & Municípios & $\begin{array}{l}\text { População } \\
\text { (hab.) }\end{array}$ & $\begin{array}{l}\text { Áreas } \\
\left(\mathbf{K m}^{2}\right)\end{array}$ \\
\hline $\mathbf{1}^{\circ}$ & Rio Branco & 413.418 & $8.835,154$ \\
\hline $\mathbf{2}^{\circ}$ & $\begin{array}{l}\text { Cruzeiro do Sul } \\
\text { (AC) }\end{array}$ & $78.507,00$ & $8.779,391$ \\
\hline $\mathbf{3}^{\circ}$ & $\begin{array}{l}\text { Sena Madureira } \\
\text { (AC) }\end{array}$ & $38.029,00$ & $23.751,494$ \\
\hline $\mathbf{4}^{\circ}$ & Tarauacá (AC) & $35.590,00$ & $20.171,053$ \\
\hline $\mathbf{5}^{\circ}$ & Feijó (AC) & $32.412,00$ & $27.974,890$ \\
\hline
\end{tabular}

O clima é classificado como equatorial quente e úmido: com estação seca (popularmente denominada de verão amazônico), período compreendido entre junho e setembro. Segundo a classificação de Köppen (1948), o clima da região está classificado como pertencente ao grupo AM, com chuvas do tipo monçônico, temperatura mínima média superior a $18^{\circ} \mathrm{C}$ e uma estação seca de pequena duração, que não influencia significativamente a cobertura vegetal. Sousa (2019) afirma que a precipitação média anual da região é de $2.012 \mathrm{~mm}$, enquanto que a temperatura média anual é de $25,46^{\circ} \mathrm{C}$ e umidade relativa média de $87,4 \%$.

\subsection{Dados e Métodos}

Os dados epidemiológicos foram coletados junto ao banco de dados do Departamento de Informática do Sistema Único de Saúde (DATASUS). Em relação aos registros de internações hospitalares por dengue (CID 10 - A90), as informações são oriundas do Sistema de Informações Hospitalares do Sistema Único de Saúde (SIH/SUS), gerido pelo Ministério da Saúde, por meio da Secretaria de Assistência à Saúde, em conjunto com as Secretarias Estaduais de Saúde e as Secretarias Municipais de Saúde, e processados pelo DATASUS da Secretaria Executiva do Ministério da Saúde. As informações disponíveis no DATASUS eram quantitativos mensais de internações para o período de 2008 a 2018.

Para que seja possível fazer a associação entre clima e saúde é necessário que os dados estejam na mesma escala temporal. Para isso, foram utilizados dados mensais de temperatura máxima média, precipitação pluvial e umidade relativa do ar da estação meteorológica de referência de Rio Branco ( $\mathrm{n}^{\circ}$ 82915), para o mesmo período que foram disponibilizados os dados de internação (2008-2018). As séries históricas completas dos dados são gerenciadas e disponibilizadas pelo Banco de Dados Meteorológicos para Ensino e Pesquisa (BDMEP), pertencente ao Instituto Nacional de Meteorologia (INMET). Com isso, pôde-se avaliar o histórico de internações anuais por dengue, pelo teste de Mann-Kendall, sugerido pela Organização Meteorológica Mundial para avaliação de tendências em séries temporais (MANN, 1945; KENDALL, 1975).

O Teste de Mann-Kendall é um método robusto, sequencial e não paramétrico utilizado para determinar se determinada série de dados possui uma tendência temporal de alteração estatisticamente significativa. Outra vantagem deste método é o fato de ser pouco influenciado por mudanças abruptas ou séries não homogêneas (ZHANG et al., 2009).

A estatística do teste é dada pela equação (1):

$$
s=\sum_{i=2}^{n} \sum_{j=1}^{i-1} \operatorname{sinal}(x i-x j)
$$

Onde a variável estatística $\mathrm{S}$, para uma série de $\mathrm{n}$ dados do teste de Mann-Kendall é calculada a partir da somatória dos sinais (sgn) da diferença, par a par, de todos valores da série (xi) em relação aos valores que a eles são futuros $\left(x_{j}\right)$. $O$ sinal $\left(x_{i}-x_{j}\right)$ é igual a -1 para $\left(\mathrm{x}_{\mathrm{i}}-\mathrm{x}_{\mathrm{j}}\right)<0,0$ para $\left(\mathrm{x}_{\mathrm{i}}-\mathrm{x}_{\mathrm{j}}\right)=0$, e 1 para $\left(\mathrm{x}_{\mathrm{i}}-\mathrm{x}_{\mathrm{j}}\right)>$ 0 .

Kendall (1975) mostrou que $S$ é normalmente distribuída com média $\mathrm{E}(\mathrm{S})$ e variância $\operatorname{Var}(\mathrm{S})$, que para uma situação na qual podem ocorrer valores iguais de $\mathrm{x}$, são calculadas pela equação (2):

$$
\operatorname{Var}[S]=\frac{n(n-1)(2 n+5)-\sum_{i-1}^{q}(t p-1)(2 t p-5)}{18}
$$

Onde n é o número de observações, caso a série tenha grupos com observações iguais; sendo que p é o número de grupos com observações iguais e t i é o número de dados com valores iguais em certo grupo i (por exemplo, uma série histórica com três valores iguais entre si teria 1 repetição de extensão igual a 3 , ou ti $=1$ e i = 3). (LIRA, et al., 2020).

Já o teste estatístico parametrizado ( $\mathrm{Z}_{\mathrm{MK}}$ ), é computado pela 
seguinte equação (3):

$$
\mathrm{ZMK}=\left\{\begin{array}{c}
\frac{S-1}{\sqrt{\operatorname{Var}[S]}} \\
0 \\
\frac{S+1}{\sqrt{\operatorname{Var}[S]}}
\end{array}\right.
$$

Para $S>0$; Para $S=0$; Para $S<0$.

A presença de uma tendência estatisticamente significativa é avaliada, usando-se o valor de Z. Tal estatística é empregada para testar a hipótese nula, isto é: que nenhuma tendência existe. Um valor positivo de $\mathrm{Z}_{\mathrm{MK}}$ indica um aumento da tendência e, quando negativa, aponta tendência decrescente. Para testar a tendência monotônica crescente ou decrescente no nível de significância de $\mathrm{p}$, a hipótese nula será rejeitada, se o valor absoluto de $\mathrm{Z}$ for maior

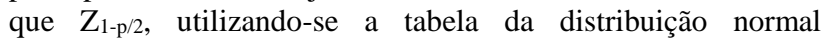
cumulativa padrão. Para o nível de significância de $5 \%$ o valor de $\mathrm{Z} / 2$ é 1.96. Os valores positivos de $\mathrm{Z}$ indicam tendências crescentes, enquanto que os valores negativos indicam tendências decrescentes (MENEZES; FERNANDES, 2016).

A partir de uma análise preliminar dos dados, foram avaliadas a associação e o tempo de resposta entre as variáveis e o número de internações por Dengue em Rio Branco. O método utilizado foi a correlação cruzada, que mede a similaridade entre os sinais de duas variáveis, em função de um atraso aplicado a uma delas. Essa técnica, que permite a detecção do grau e do momento onde as duas variáveis se correlacionam, reflete como os dois processos estão correlacionados (GOMES et al., 2018). Sua representação gráfica determina, se uma série de dados conduz outra série, e até que ponto. $\mathrm{O}$ estimador da função correlação cruzada é calculado pela seguinte equação (4):

$$
\hat{\rho} x y(h)=\frac{\sum_{t=1}^{n-h}(x t+h-\bar{x})(y t-\bar{y})}{n^{-1} \sum_{n=1}^{n}(x t-\bar{x})^{2}\left(\sum_{t=1}^{n} y t-\bar{y}\right)^{2}}
$$

Onde, $\boldsymbol{X}_{\boldsymbol{t}}$ e $\boldsymbol{Y}_{\boldsymbol{t}}$ são as séries temporais; x e y são as médias; h é o coeficiente de defasagem entre as séries e; n é o número de observações. O método estatístico foi realizado com o auxílio do software estatístico livre R versão 3.5.1.

\section{RESULTADOS E DISCUSSÃO}

Durante o período analisado, os meses com mais registros mensais de dengue são os de janeiro a março, chegando ao total de $60 \%$ dos casos, com destaque para o mês de fevereiro. Quanto às taxas anuais, os anos de 2009 e 2010 foram os que mais apresentaram internações, onde $65 \%$ dos casos de dengue foram registrados nesse intervalo.

A Figura 2 mostra a distribuição das internações por dengue no período de 2008 a 2018. Os anos de 2009, 2010 e 2011 apresentaram os maiores índices de internações, com uma notável diminuição dos casos a partir de 2012. Pelo teste de Mann Kendall, tendências de decréscimo com significância estatística (p-valor 000,1) foram encontradas.

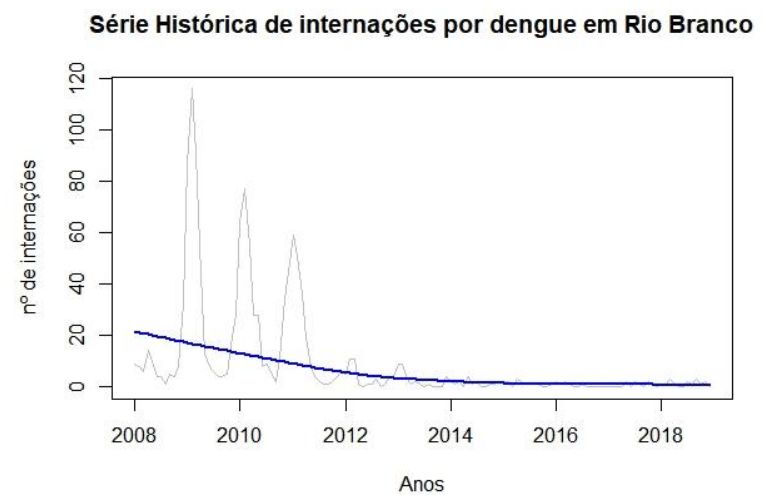

Figura 2 - Série histórica das internações por dengue e sua respectiva tendência. Fonte: SIH/SUS (2019). Organização: os autores (2021).

Apesar dos valores terem diminuídos, não significa que a doença esteja mitigando-se. O Sistema de Informação de Agravos de Notificação, durante o período de 2008 a 2018 registrou cerca de 72.000 casos de notificações da doença. Logo, as internações diminuíram, mas o vetor ainda opera (SINAN, 2019).

A precipitação média do período analisado (Figura 3) revela o maior (menor) índice de chuva no mês de janeiro (julho). Conforme mostrado por Baracho et al., (2014), a influência pluviométrica na patologia é geralmente dada como significativa, principalmente nos casos ocorridos durante verão, onde o índice pluviométrico é intensificado. Destaca-se também o mês de janeiro como o de maior variabilidade e a presença de valores discrepantes superiores em fevereiro, junho, setembro e novembro.

Preciptação média mensal em Rio Branco

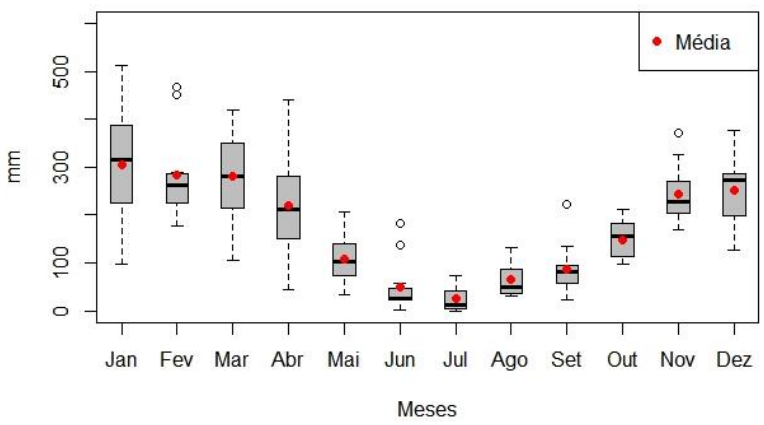

Figura 3 - Precipitação média mensal para o município de Rio Branco, no perído de 2008 a 2018. Fonte: BDMEP/INMET (2019). Organização: os autores (2021).

A temperatura influencia diretamente no desenvolvimento do ciclo do mosquito, cuja variação é de 5 a 7 dias. Logo, para o seu desenvolvimento são necessárias condições ambientais adequadas. Segundo Pacheco et al (2017), a variação da temperatura ideal para o ciclo biológico do Aedes Aegypti é de $22^{\circ} \mathrm{C}$ a $30^{\circ} \mathrm{C}$. Por outro lado, temperaturas abaixo das citadas 
aumentam o tempo estimado para o desenvolvimento do mosquito.

Para a temperatura, são apresentados dois gráficos de temperaturas médias máximas e mínima do ar (Figuras 4-5). Na Figura 4 , o menor valor $\left(31^{\circ} \mathrm{C}\right)$ ocorreu no mês de junho, enquanto o maior foi observado em setembro $\left(34^{\circ} \mathrm{C}\right)$. As médias de temperatura máxima da região apresentam alta variabilidade nos meses de junho a agosto, transição entre os períodos de menores para os maiores valores de temperatura.

\section{Temperatura máxima média mensal em Rio Branco}

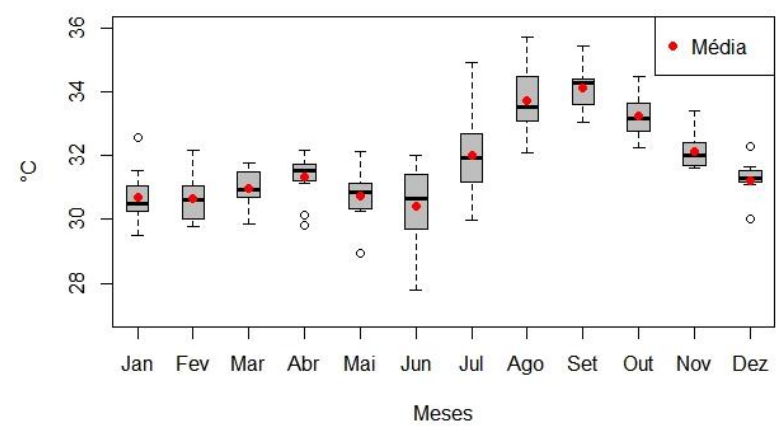

Figura 4 - Temperatura média máxima mensal no munícipo de Rio Branco, durante o período 2008-2018. Fonte: BDMEP/INMET (2019). Organização: os autores (2021).

Ao comparar os gráficos de precipitação e temperatura média máxima mensal (Figuras 3 e 4), pode-se notar que os meses com temperaturas médias máximas mais elevadas são os que apresentam os menores índices pluviométricos. Já o gráfico de temperatura média mínima mensal (Figura 5), mostra que o menor valor de temperatura foi registrado em julho, em torno de $18^{\circ} \mathrm{C}$ e, o maior foi obtido em novembro, em torno de $24^{\circ} \mathrm{C}$. É importante notar que a variabilidade da temperatura mínima é inferior à observada nos valores da temperatura máxima. Destacase também que valores discrepantes superiores foram detectados nos meses de janeiro, julho e setembro.

Temperatura mínima média mensal em Rio Branco

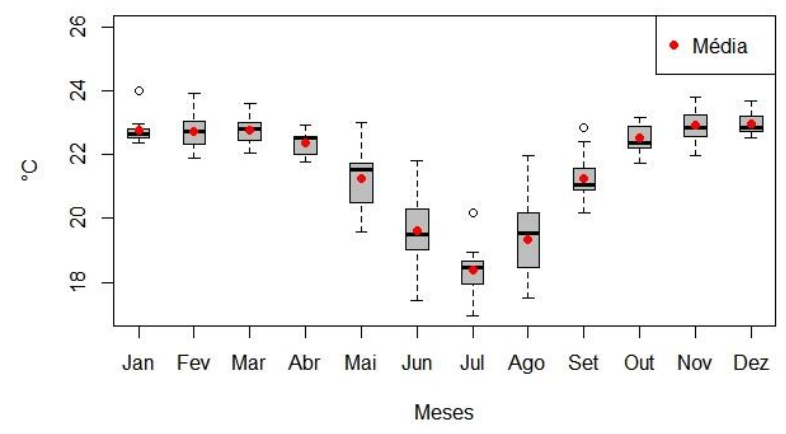

Figura 5 - Temperatura média mínima mensal no município de Rio Branco, durante o período 2008-2018. Fonte: BDMEP/INMET (2019). Organização: os autores (2021).
Segundo Baracho et al (2014), o Aedes Aegypti tem uma grande potencialidade de se desenvolver, quando a umidade relativa do ar se apresenta na faixa compreendida entre $70 \%$ e $100 \%$, onde o desenvolvimento do mosquito será satisfatório para todas as fases do ciclo. Ao analisarmos a umidade relativa mensal (Figura 6), observa-se que a umidade apresenta índices elevados, com a menor maior porcentagem detectada no mês de agosto (74\%), enquanto a maior obtida em janeiro (90\%), o que apresenta condições favoráveis para proliferação do mosquito.

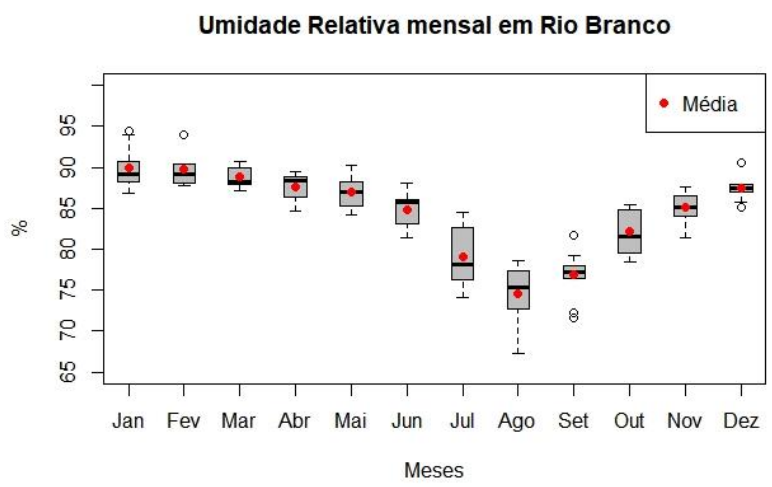

Figura 6 - Umidade relativa mensal no município de Rio Branco, durante o período 2008-2018. Fonte: BDMEP/INMET (2019). Organização: os autores (2021).

O gráfico de internações mensais no município (Figura 7) mostra os valores de morbidade por dengue. Evidencia-se que os casos de internações são maiores durante o período de novembro a abril, e diminuem a partir do mês de maio até outubro. Os meses com maiores índices de internações coincidem com o cenário atmosférico favorável para a proliferação do mosquito, na presença de intensificações da precipitação e umidade relativa, porém com temperaturas médias do ar mais amenas.

Internações mensais em Rio Branco

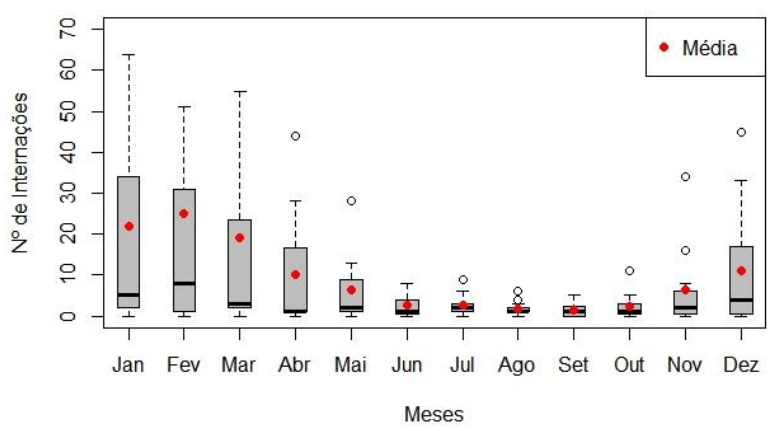

Figura 7 - Internações mensais no munícipio de Rio Branco, durante o período 2008-2018. Fonte:SIH/SUS (2019). Organização: os autores (2021).

Viana et al. (2013) também relacionaram o aumento dos casos do vírus com a precipitação. Entretanto, as Figuras 3-7 mostram 
que as análises dos fatores meteorológicos significativos associados à transmissão da dengue podem variar, dependendo das condições climáticas da região.

Nos meses em que se obtiveram os maiores índices de casos da patologia, a temperatura variou de $30^{\circ} \mathrm{C}$ a $32^{\circ} \mathrm{C}$, cujos limiares são superiores aos propostos na literatura. Isso sugere uma adaptação do mosquito ao passar dos anos, resistindo às temperaturas maiores. Adicionalmente, observa-se que nos meses em que a temperatura decai, as taxas de casos de dengue também diminuem, sinalizando uma relação direta. Entretanto, em geral, tanto o verão quanto o inverno apresentaram altas temperaturas, que são adequadas para a proliferação do vetor, (FERREIRA et al., 2018). Segundo Pacheco et al. (2017), a diminuição dos casos do vírus não exclui a circulação do mesmo entre a população.

Ao analisar visualmente a precipitação, é possível notar que há uma relação entre os índices de precipitação e os casos de dengue. Os casos registrados entre os meses dezembro a abril, onde apresentaram altos índices pluviométricos mensais, foram os meses, onde ocorreram os maiores números de casos de dengue confirmados no local de estudo. Baracho et al. (2014) cita que esta situação ocorre devido ao favorecimento de acúmulo de água em recipientes artificiais nas residências que acabam se tornando criadouros do mosquito Aedes Aegypti. Ao observar a umidade relativa do ar, pode-se verificar que os picos de umidade coincidem com os picos de morbidade por dengue na população (Figura 6). A região apresentou a porcentagem da taxa de umidade alta para todo o ano, onde os valores mais altos coincidem com os meses de maior incidência da patologia.

De acordo com Ferreira et al. (2018), as taxas de pluviosidade e as temperaturas elevadas contribuem para o aumento do número de criadouros e casos de dengue. Em períodos secos e de baixas temperaturas, há diminuição no número de vetores, mas não é suficiente para cessar a transmissão da doença, devido ao comportamento hematofágico do vetor que ocorre por todo ano. Horta et al (2014) mostraram que modelos com base em variáveis climáticas que consideram o intervalo entre pluviosidade, temperatura do ar e dengue podem ser úteis em programas de controle de dengue em países tropicais. Em termos práticos, demonstrou-se a partir da técnica de correlação cruzada que apenas com a precipitação e os dados de internação, não foi possível verificar o tempo de associação (Figura 8).

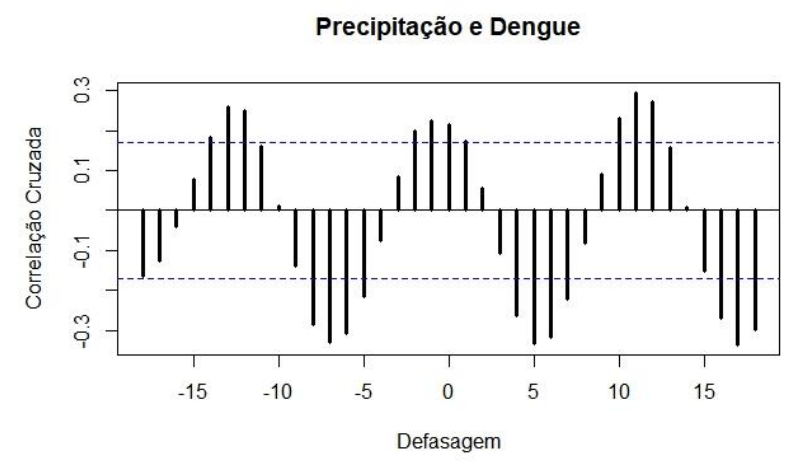

Figura 8 - Correlação cruzada entre a preciptação e os casos de dengue no município de Rio Branco, durante o período 20082018. Fonte:SIH/SUS (2019); BDMEP/INMET (2019). Organização: os autores (2021).

É possível verificar associação entre a temperatura máxima e os casos de dengue, sinalizando que a sazonalidade presente é fundamental para a visualização da correlação. Acredita-se que o auxílio na proliferação dos mosquitos contribui para a ocorrência das internações no período subsequente. $O$ que pode ser confirmado com o Lag 7 na correlação cruzada entre temperatura máxima do ar e os casos de dengue (Figura 9).

Temperatura Máxima e Dengue

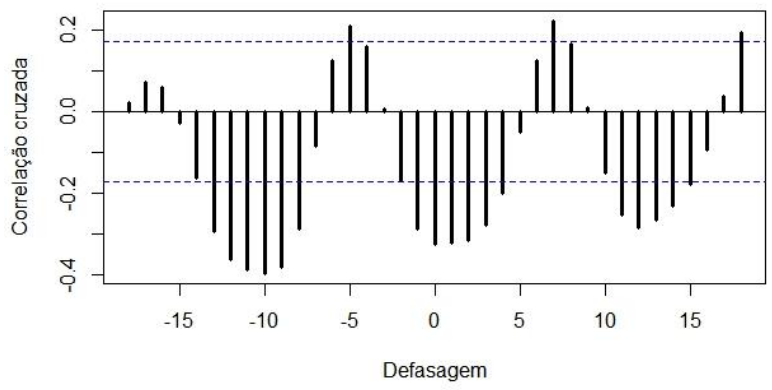

Figura 9 - Correlação cruzada entre a temperatura máxima do ar e os casos de dengue no município de Rio Branco, durante o período 2008-2018. Fonte:SIH/SUS (2019); BDMEP/INMET (2019). Organização: os autores (2021). 
Já a temperatura mínima, apresentou associação inversa com os lags 3, 4 e 5 na correlação cruzada entre temperatura mínima e os casos de dengue (Figura 10), sinalizando que alterações na temperatura mínima contribuem para com a diminuição das internações por dengue, com defasagem de 3 a 5 meses.

Temperatura Mínima e Dengue

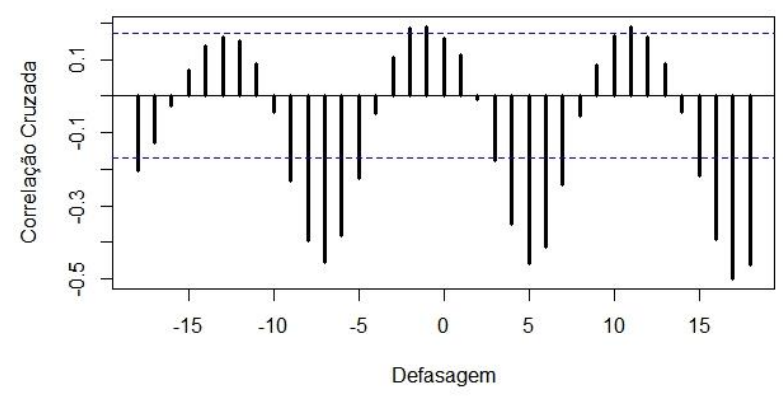

Figura 10 - Correlação cruzada entre a temperatura mínima e os casos de dengue no município de Rio Branco, durante o período de 2008 a 2018. Fonte:SIH/SUS (2019); BDMEP/INMET (2019). Organização: Os autores (2021).

A partir da correlação cruzada (Figura 11), observa-se que o sinal da sazonalidade é predominante entre a umidade relativa do ar e as internações por dengue. Portanto, sugere-se que o ciclo sazonal da variável meteorológica influencia diretamente nas internações, conforme às associações significativas ao longo dos períodos. Esses resultados corroboram com aqueles obtidos por Ramalho (2008), que mostra a influência da alta taxa de umidade relativa do ar nos padrões de alimentação, no aumento da taxa de reprodução e na maior longevidade do Aedes Aegypti.

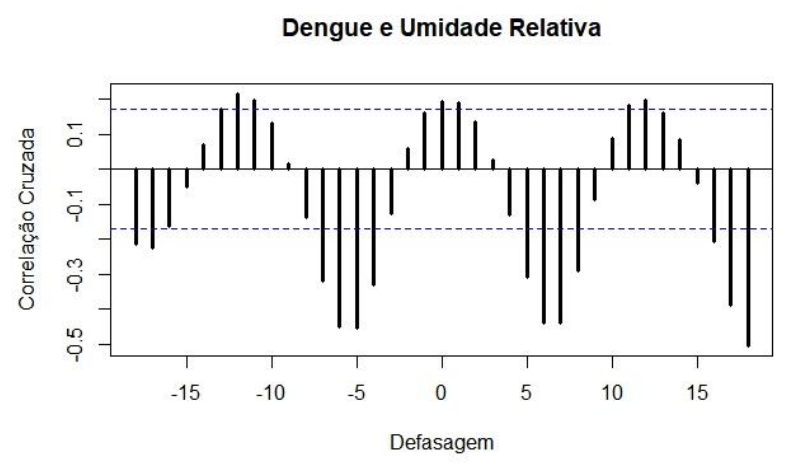

Figura 11 - Correlação cruzada entre a umidade relativa do ar e os casos de dengue no município de Rio Branco, no período de 2008 a 2018. Fonte:SIH/SUS (2019); BDMEP/INMET (2019). Organização: Os autores (2021).

\section{CONSIDERAÇÕES FINAIS}

Baseado nas associações entre as variáveis meteorológicas e os casos de internações por dengue no munícipio de Rio Branco, durante o período 2008 a 2018, este estudo avaliou estatisticamente as correlações das variáveis da precipitação pluviométrica, umidade relativa do ar, temperaturas mínimas e máximas do ar com os casos de internações registrados pelo DATASUS.

Foram encontradas associações siginifcativas entre as variáveis meteorológicas e as ocorrências de internações por dengue em Rio Branco, no Acre. O cenário mostrou-se favorável em condições de altos valores de precipitação pluvial e umidade relativa do ar, porém com temperaturas mais amenas. É importante destacar, que houve uma diminuição nos registros de internações, cuja tendência decrescente significativa foi de $61 \%$ dos casos, no intervalo de 2012 a 2018, mas sem evidências de que a doença esteja diminuindo, visto que há evidências do aumento de registros de notificações pelo SINAN para o mesmo período.

Embora as avaliações priorizem o munícipio de Rio Branco (localidade de maior proliferação do Aedes Aegypti no Brasil), os resultados cooperam com pesquisas anteriormente realizadas em outras regiões, por referenciar valores atípicos da temperatura máxima do ar favoráveis ao desenvolvimento do mosquito, com limiares variando de $30^{\circ} \mathrm{C}$ a $32^{\circ} \mathrm{C}$. Além disso, a combinação das variáveis meteorológicas de pluviosidade e temperatura do ar pode ser útil nos programas de controle de dengue em regiões tropicais.

\section{REFERÊNCIAS}

ACRE. Governo do Estado do Acre. Zoneamento EcológicoEconômico do Estado do Acre, Fase II (Escala 1:250.000): Documento Síntese. 2. Ed. Rio Branco: SEMA, 2010. 356p.

ALMEIDA, L. S.; COTA, A. L. S.; RODRIGUES, D. F. Saneamento, Arboviroses e Determinantes Ambientais: impactos na saúde urbana. Ciência \& Saúde Coletiva, v. 25, n. $10,3857-3868,2020$.

ALVES, E. D. L. MENDONÇA, F.; DANNI-OLIVEIRA, I. M. Climatologia: noções básicas e climas do Brasil. Sociedade \& Natureza, v. 22, n. 3, 206 p. 2011.

ANDRADE, I. S. Influência de Elementos Meteorológicos nos Casos de Cólera, Dengue e Meningite no Estado da Paraíba. Campina Grande, 2003. 105f. Dissertação (Mestrado em Biometeorologia). Programa de Pós-Graduação em Meteorologia, Universidade Federal de Campina Grande, Campina Grande-PB, 2003.

BARACHO, R. C. M. Influência de variáveis meteorológicas sobre a incidência dos casos de dengue no município de areia-pb. Campina Grande, 2013. 31 f. Tese (Graduação em Biologia), Universidade Federal da Paraíba, Campina Grande-PB, 2013.

BARACHO, R. C. M.; FILHO A.I; GONÇALVES A.; NUNES S.T.S; BORGES P.F. A influência climática na proliferação da dengue na cidade de Areia, Paraíba. Gaia Scientia, v. 8, n.1, 65-73, 2014.

BARBOSA, Bárbara Charine Machado, Arbovírus emergentes no Brasil e seu risco de transmissão por transfusão sanguínea: 
uma revisão da literature. Belo Horizonte, 2017. 69f. Monografia. Programa de Pós-Graduação em Microbiologia do Instituto de Ciências Biológicas, Universidade Federal de Minas Gerais, Belo Horizonte-MG, 2017.

BARROS NETO, B.; SCARMINIO, I. S.; BRUNS, R. E. Como Fazer Experimentos: Pesquisa e Desenvolvimento na Ciência e na Indústria. Campinas: Editora da UNICAMP, 2001. $480 \mathrm{p}$.

BRASIL. Ministério da Saúde. Secretaria de Vigilância em Saúde. Guia de Vigilância Epidemiológica. 7 ed. rev Brasília: Ministério da Saúde, 2009. 816 p.

BRASIL. Ministério da Saúde. Secretaria de Vigilância em Saúde. In Sistema de Informação de Agravos e Notificação. Disponível em: http://sinan.saude.gov.br/sinan/login/login.jsf. Acesso em: 02/12/2019.

CONASS. Conselho Nacional de Secretários de Saúde. Ministério da Saúde alerta para aumento de 149\% dos casos de dengue no país. Organização: Alexandre Penido. 2019.

COSTA, E. A. P. de A.; SANTOS, E. M. de M.; CORREIA, J. C.; ALBUQUERQUE, C. M. R. Impact of small variations in temperature and humidity on the reproductive activity and survival of Aedes aegypti (Diptera, Culicidae). Revista Brasileira de Entomologia, v. 54, n. 3, 488-493, 2010.

DA SILVA, J. M. C.; RYLANDS, ANTHONY B.; DA FONSECA, GUSTAVO A.B. The fate of the Amazonian areas of endemism. In: Conservation Biology. v. 19, n. 3, 689-694, 2010.

DATASUS. Ministério da Saúde, Brasil, 2018. In Tabnet. Disponivel em: http://datasus.saude.gov.br/informacoes-desaude/tabnet. Acesso em: 19/11/2019.

DUARTE, A. F.; MASCARENHAS, M. D. M. Manifestações do bioclima do Acre sobre a saúde humana no contexto socioeconômico da Amazônia. Amazônia Ciência $e$ Desenvolvimento. v. 3, n. 5, 149-162, 2007.

FERREIRA, A. C.; CHIARAVALLOTI NETO, F.; MONDINI, A.. Dengue in Araraquara, state of São Paulo: epidemiology, climate and aedes aegypti infestation. Revista de Saúde Pública, v. 52, p. 18, 26, 2018.

FUNASA. Dengue instruções para pessoal de combate ao vetor: manual de normas técnicas. Brasília: Ministério da Saúde: Fundação Nacional de Saúde, 2001. 84 p.

GABRIEL, A. F. B.; ABE, K. C.; GUIMARÃES, M. de P.; MIRAGLIA, S. G. El K. Avaliação de impacto à saúde da incidência de dengue associada à pluviosidade no município de Ribeirão Preto, São Paulo. Cadernos Saúde Coletiva, v. 26, n. 4, 446-452, 2018. doi: http://dx.doi.org/10.1590/1414$462 \times 201800040119$.
GOMES, A. C. S.; CONSTANTINO, S. M. H.; LUCIO, P. S. Dynamic Regression Model for Evaluating the Association Between Atmospheric Conditions and Deaths due to Respiratory Diseases in São Paulo, Brazil. Revista Brasileira de Meteorolologia, v. 33, n. 1, 1-10, 2018. doi: https://doi.org/10.1590/0102-7786331001.

GOMES, A. F.; NOBRE, A. A.; CRUZ, O. G. Temporal analysis of the relationship between dengue and meteorological variables in the city of Rio de Janeiro, Brazil, 20012009. Cad. Saúde Pública, v. 28, n. 11, 2189-2197, 2012. doi: http://dx.doi.org/10.1590/S0102-311X2012001100018.

GUZMAN, M. G.; HARRIS, E. Dengue. The Lancet, v. 385, n. 9966, 4453-465, 2015.

HALES S.; De WET N.; MAINDONALD J.; WOODWARD A. Potential effect of population and climate changes on global distribution of dengue fever: an empirical model. The Lancet, v. 360, n. 9336, 830-834. 2002 . doi: 10.1016/S01406736(02)09964-6.

HORTA, M. A.; BRUNIERA, R.; KER, F.; CATITA, C.; FERREIRA, A. P. Temporal relationship between environmental factors and the occurrence of dengue fever. International Journal of Environmental Health Research, v. 24, n. 5, 471-481, 2014. doi: http://dx.doi.org/10.1080/09603123.2013.865713

IBGE, Instituto Brasileiro de Geografia e Estatística. Brasília: Ministério do Planejamento, Orçamento e Gestão. Disponível em:http:// mapas.ibge.gov.br/clima/viewer.htm. Acesso em: 19/11/2019.

IBGE. Instituto Brasileiro de Geografia e Estatística. Cidades. Rio Branco. Disponível em: http://ibge.gov.br/cidadesat. Acesso: 20/11/2019.

IBGE. Instituto Brasileiro de Geografia e Estatística. In Portal de Mapas. Disponível em: https://portaldemapas.ibge.gov.br/portal.php\#mapa201449. Acesso em: 20/11/2019.

INMET, Instituto Nacional de Meteorologia. BDMEP - Banco de Dados Meteorológicos para Ensino e Pesquisa. Disponível em: https://bdmep.inmet.gov.br.

KENDALL, M. G. Rank correlation methods. Griffin, London, 1975. 202 p.

KOPPEN, W. Climatologia:com um estúdio de los climas de la tierra. México: Fondo de Cultura Economica, 478p. 1948.

LIMA, E. A.; FIRMINO, J. L. N; GOMES FILHO, M. F. A relação da previsão da precipitação pluviométrica e casos de dengue nos estados de Alagoas e Paraíba nordeste do Brasil. Revista brasileira de meteorologia, v. 23, n.3, 264269, 2008. doi: https://doi.org/10.1590/S010277862008000300001 
LIRA, B., LOPES, L., CHAVES, J., SANTANA, L., \& FERNANDES, L. Identificação de Homogeneidade, Tendência e Magnitude da Precipitação em Belém (Pará) entre 1968 e 2018. Anuário do Instituto de Geociências, v. 43, n.4, 426-439, 2020.

LOPES, R. A. Monitoramento dos casos de dengue e a relação com a desigualdade sócio-espacial no Distrito Federal. Brasília, 2015. 61f. Monografia (Bacharelado em Saúde Coletiva). Universidade de Brasília, Brasília-DF, 2015.

MENDONÇA F. A.; SOUZA A. V.; DUTRA D. A..; Saúde pública, urbanização e dengue no Brasil. Sociedade $e$ natureza, v. 21, n. 3, 257-269, 2009. doi: http://dx.doi.org/10.1590/s1982-45132009000300003.

MESQUITA, R. C; DUARTE, A. F. Diferenças na climatologia das chuvas entre as regiões leste e oeste do estado do Acre. In: congresso de estudantes e bolsistas do experimento LBA, Anais. Manaus, 2005.

MONDINI, A.; DE MORAES BRONZONI, R.V.; NUNES, S.H.P.; NETO, F.C.; MASSAD, E.; ALONSO, W.J., et al. Spatio-temporal tracking and phylodynamics of an urban dengue 3 outbreak in Sao Paulo, Brazil. PLoS Negl. Trop. Dis, v. 3, n. 5, 15p, 2009. doi: https://doi.org/10.1371/journal.pntd.0000448.

NETO, T. S. C., RAMIREZ, M. T. P., GALINDO, V. R., HERCULANO, L. F. S., CAMPELlO, M. V. M. Levantamento de potenciais criadouros de Aedes aegypti no Campus do Itaperi da Universidade Estadual do Ceará. Medicina Veterinária (UFRPE), v. 13, n. 1, 43-48, 2019. doi: 10.26605/medvet-v13n1-2608.

NOGUEIRA, R. M. R.; MIAGOSTOVICH, M. P.; SCHATZMAYR, H. G. Molecular epidemiology of dengue viruses in Brazil. Caderno de Saúde Pública, v. 16, n.1, 205211 , 2000. doi: https://doi.org/10.1590/S0102$311 X 2000000100021$

PACHECO I. C. S.; CARVALHO A. M. A.; PONTES E. R. J. C.; SILVA M. G. Relação entre condições climáticas e incidência de dengue no município de Campo Grande, MS. Multitemas, v. 22, n. 51, 236-252, 2017. doi: https://doi.org/10.20435/multi.v22i51.1123;

RAMALHO W.M. Influência do regime de chuuvas na ocorrência do dengue em municipios brasileiros, 2002 a 2006. Salvador, 2008. Dissertação (mestrado), Instituto de Saúde Coletiva, Universidade Federal da Bahia, SalvadorBA, 2008.

SALVIANO, M. F.; GROPPO, J. D.; PELLEGRINO, G. Q. Análise de Tendências em Dados de Precipitação e Temperatura no Brasil. Revista Brasileira de Meteorologia, v.31, n.1, 64-73, 2016. doi:http://dx.doi.org/10.1590/0102778620150003

SCHATZMAYR H. G. Dengue situation in Brazil by year 2000. Memórias do Instituto Oswaldo Cruz, v. 95, n. 1, 179-181,
$2000 . \quad$ doi: http://dx.doi.org/10.1590/s007402762000000700030 .

SILVA R. A.; SILVA V.P.R.; CAVALCANTI E.P.; SANTOS D.N. Estudo da variabilidade da radiação solar no Nordeste do Brasil. Revista Brasileira de Engenharia Agrícola e Ambiental, v. 14, n. 5, 501-509, 2010. doi: http://dx.doi.org/10.1590/s1415-43662010000500007.

SILVA, A. M.; SILVA, R. M.; ALMEIDA.; C. A. P.; CHAVES, J. J. S. Modelagem geoestatística dos casos de dengue e da variação termopluviométrica em João Pessoa, Brasil. Sociedade e Natureza, v. 27, n. 1, 157-169, 2015.

SILVA, V. DE P. R. DA; CAVALCANTI, E. P.; BRAGA, C. C.; AZEVEDO, P. V. Evaluating trends in solar radiation based on data fields from the NCEP/NACR reanalysis and measurements, 7th EMS Annual Meeting/8th ECAM. In: El Escorial, Madrid Anais... Madrid: European Meteorogical Society, 2007.

SIQUEIRA, I. S.; QUEIROZ J. C. B.; AMIN, M. M.; CÂMARA, R. K. C. A Relação da Incidência de Casos de Dengue com a Precipitação na Área Urbana de Belém-PA, 2007 a 2011, Através de Modelos Multivariados de Séries Temporais. Revista brasileira de Meteorologia, v. 33, n. 2, 380-389, 2018. doi: http://dx.doi.org/10.1590/0102-7786332010.

SIQUEIRA, J.A.S.; ALENCAR, L.D.; CAVALCANTE, L.P.S. Impactos da urbanização desordenada na saúde pública: leptospirose e infraestrutura urbana. Revista Eletrônica Polêmica, v.13, n.1, 1006-1020, 2014.

SOUSA, J. W. Características climáticas do município de Rio Branco, Acre, período de 1990-2019. Scientia Naturalis, v. 2, n. 2, 723-740, 2020.

SOUSA, N. M. N. Influência de variáveis meteorológicas sobre a incidência do Dengue, Meningite e Pneumônia em João Pessoa-PB. Revista Brasileira de Meteorologia, v.22, n.2, 183-192, 2005.

SOUZA-SANTOS, Reinaldo. Fatores associados à ocorrência de formas imaturas de Aedes aegypti na Ilha do Governador, Rio de Janeiro, Brasil. Revista da Sociedade Brasileira de Medicina Tropical, v.32, n.4, 373-382, 1999. doi: https://doi.org/10.1590/S0037-86821999000400007.

VIANA, D. V.; IGNOTTI, E. A ocorrência da dengue e variações meteorológicas no Brasil: revisão sistemática. Revista Brasileira de Epidemiologia, v. 16, n. 2, 240-256, 2013. doi: http://dx.doi.org/10.1590/s1415790x2013000200002

ZHANG, W; YAN, Y; ZHENG, J; LI, L; DONG, X; CAI, H. Temporal and spatial variability of annual extreme water level in the Pearl River Delta region, China. Global and Planetary Change. v. 69, n. 1-2, 35-47, 2009. doi: http://dx.doi.org/10.1016/j.gloplacha.2009.07.003. 


\section{AGRADECIMENTOS}

À Universidade Federal do Oeste do Pará (UFOPA) e ao Instituto de Engenharia e Geociências (IEG) pelo apoio.

Recebido em: 31/03/2021

Aceito para publicação em: 20/06/2021 